\title{
Comparative Analysis between Experimental and Numerical Model resultsof a Jetin a Shallow Water Tank
}

\author{
Robles L., Jose I., Mejia A., Victor \\ Environmental HydraulicDepartment \\ IMTA \\ Morelos.Mexico \\ irobles@tlaloc.imta.mx
}

\author{
Palacio P., Arturo, Rodriguez V., Alejandro \\ Engineering Insititute of the \\ UNAM \\ Mexico, D.F., Mexico \\ APalacioP@iingen.unam.mx
}

\begin{abstract}
In this work a comparative analysis of a turbulent jet experiment produced by the injection of dyed water into a shallow tank filled with water of the same density and the results of a numerical modelare presented. Dye was injected with the source fluid as a tracer. The concentration of the dye in the shallow turbulent flow was determined using a video imaging technique.The present laboratory experiments were conducted in a tank of small depth, and it is significantly wide to avoid the effect of the side walls. The space between the parallel walls of the tank can be varied during the experiments. The large-scale turbulent flow in the water sheet between the walls of the tank is confined to essentially two-dimensional motion. The shear on the bottom of the tank is a momentum sink to be considered.A comparison of the numerical resultswith the experimental data showed a very good agreement in terms of the position reached by the jet at different times after injection is initialized. These findings are useful for turbulent modeling of the shallow shear flow and for application to the large scale heat and mass exchange processes in lagoons, lakes, the ocean and the atmosphere.
\end{abstract}

Keywords—Jets, shallow flows, turbulence, numerical models.

\section{INTRODUCTION}

Large-scale and small-scale turbulence of distinct length scales are present in rivers, lakes and oceans in order to produce heat and mass exchange processes in shallow shear flows. The large-scale turbulence produced by the horizontal shear of a jet is confined to a predominantly horizontal motion between the boundary of the free surface and the channel bed. The confinement of large scale motion to a small depth leads to two dimensional motion. Small-scale turbulence motion, on the other hand, with a large scale less than the depth of flow is three-dimensional, since it is free to move in all directions. Quasi two dimensional turbulent flow consisting of large-scale and small-scale turbulent motion has been the subject of a number of recent investigations. Rastogi and Rodi[12] proposed a depth-averaged version of the $k-\varepsilon$ model for turbulent flows of shallow depth. Booij[7] introduced a modification of the model of Rastogi and Rodi. Madsen [9] whereby he experimented with the method of the large eddy simulation.Nadaoka and Yagi[10] introduced a sub-depth scale model, using a $\mathrm{k}-\varepsilon$ model for the small-scale turbulence and computed the large eddies through direct numerical simulation. Chu and Babarutsi[2] and Babarutsi and Chu [6] introduced the concept of two-length scale modeling.

Numerous model coefficients were introduced through various modeling attempts. The assessment of the model performance depends on experimental data, but shallow shear flows of small depth are difficult to produce. The measurement of flow for small depth is often difficult in a laboratory setting.

\section{LABORATORY STUDIES}

A experimental facility must have a large lateral length scale compared with the depth, in order to properly represent these phenomena. Measurement of small depth flow is difficult, as the size of the instrumentation is often too large compared with the flow depth. Despite this difficulty, experiments on the friction effect of the transverse shear has been conducted in a laboratory setting by $\mathrm{Chu}$ and Babarutsi[1]. The transverse shear flow examined by Chu and Babarutsi[1] was produced in a mixing layer downstream of a splitter plate in a shallow open channel. Measurement of velocity and turbulent intensity in the mixing layer have shown the flow to be affected by friction. The mixing layer in shallow depth does not increase in width indefinitely with distance from the splitter plate but tends to approach a width of finite magnitude. The calculation of the bed-friction number in a mixing layer of finite depth produces a critical bed-friction number, $\overline{S_{c}}$, of about 0.08 , which is consistent with the analysis by Chu, Wu and Khayat[5].

\section{EXPERIMENTAL EQUIPMENT}

In the experimental setupherein, a tank with a large lateral length scale compared with the depth has been designed. It has a ratio L/h of 5.35 E04, approximately. Quasi two dimensional flow produced in the tank depends on both the momentum flux and the buoyancy flux of the source. In the first series of jet experiments, care was taken to ensure that the difference in water temperature betwen the source and the tank waswithin a measurable limit of 0.05 degreesCelsius (figure 1 ). It may then 


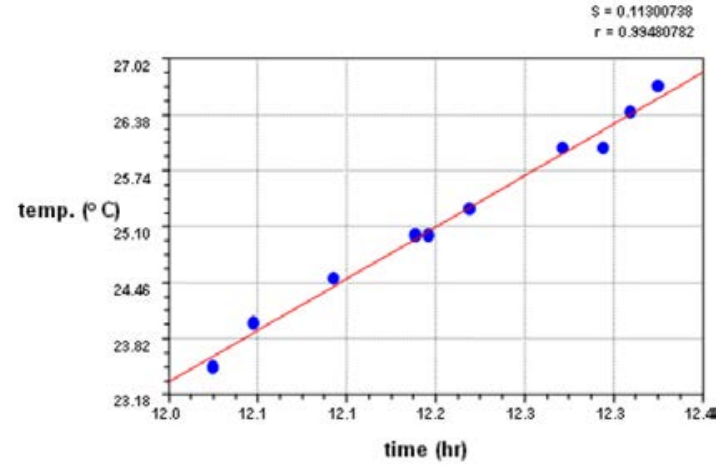

Fig. 1. Increase temperature of injection water due the bomb.

be assumed that the jet has no significant bouyant force within the tank.

The momentum flux is nearly a constant in the test TW (Wide Test). In this case, the depth of the flow $\mathrm{h}=0.044 \mathrm{~m}$ is large and the wall friction is considered to be negligible. This kind of turbulent flow is as show in figures 5 to 8.The initial development characterized by the formation of jet front which is significantly greater than the jet upstream. Upon impingement of the jet front on the edge of the tank, the jet splits up and glides along this boundary.The turbulent flow then moves upsteam along the left and right side walls.

To minimize the deflection of the walls, the tank was constructed with a double wall structure. The walls, of the inner tank were kept perfectly parallel to each other by filling both the inner and outer tank with water. Wall deflection was eliminated since the net hydrostatic pressure on the inner tank walls is zero. The outer tank walls are $1.25 \mathrm{~m}$ high and $2.45 \mathrm{~m}$ wide. The inner tank walls are $1.10 \mathrm{~m}$ and $2.35 \mathrm{~m}$, respectively. The distance between the parallel walls in the inner tank is $0.044 \mathrm{~m}(\mathrm{~W})$. Table 1 summarizes the test parameters of the experiment.

Blue dye of known concentration was used as a tracer. The turbulent flow was illuminated by back light and recorded by a video camera (SONY 3CCD) at a rate of 30 frames per second. The video images were subsequently digitized frame by frame and analyzed using a computer.

TABLE I. TEST CONDITIONS

\begin{tabular}{|l|c|c|c|}
\hline \multirow{2}{*}{ Test } & \multicolumn{3}{|c|}{ Parameters } \\
\cline { 2 - 4 } & $\boldsymbol{h}(\boldsymbol{m})$ & $\boldsymbol{Q}_{0}(\mathbf{l} / \mathbf{s})$ & $\boldsymbol{C}_{\boldsymbol{o}}(\mathbf{k g} / \mathbf{l})$ \\
\hline TW & 0.044 & 0.06898 & $0.6 \times 10^{-4}$ \\
\hline
\end{tabular}

The video images are stored as 24 BPP in BMP format. There are $640 \times 480$ pixels in a frame and each pixel has three basic colors, red, green and blue, each with values varying from 0 to 255 (table 2). The RGB values in the BMP file are proportional to the intensities of red, green and blue light through the video camera and are related to the concentration of the dye in the turbulent flow through a calibration curve (figure 2).

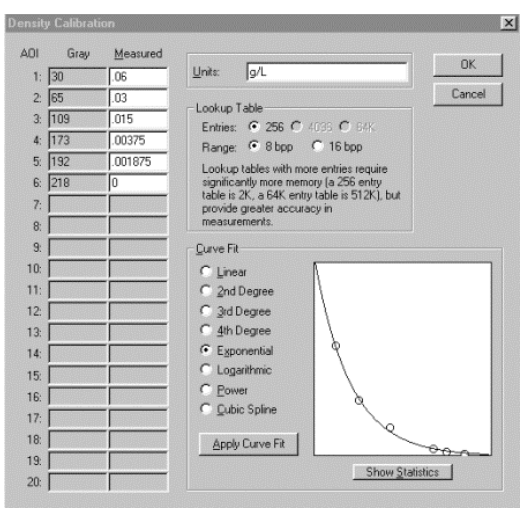

Fig. 2.Calibration curve.

The calibration of the video camera was conducted using diluted samples of known dye concentration. Video images were taken of the samples in a small plexiglass box of the same thickness as the inner tank and under the similar lighting condition as the experiment (figure 3).

TABLE II. COLOR CODING

\begin{tabular}{|c|c|c|c|}
\hline red & green & blue & result \\
\hline 0 & 0 & 0 & black \\
\hline 255 & 255 & 255 & white \\
\hline 0 & 255 & 0 & pure red \\
\hline 0 & 0 & 255 & pure blue \\
\hline $\mathrm{x}$ & $\mathrm{x}$ & $\mathrm{x}$ & any color \\
\hline
\end{tabular}

The exponential relationship is used to correlate the relative dye-concentration, $\mathrm{c} / \mathrm{c} 0$ with the $\mathrm{p}$-value.

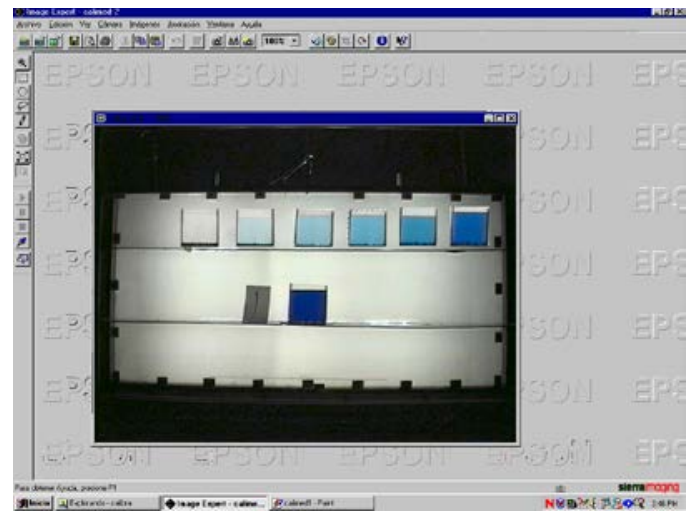

Fig.3. Diluted samples of know dye concentration.

The concentration of the dye in the turbulent flows of the jet is determined for each of the $640 \times 480$ pixels in the image file. Since the light intensity over the $1.10 \mathrm{~m}$ x $2.35 \mathrm{~m}$ area of the tank is not exactly uniform, the dye concentration is determined thought the change in light intensity relative to the light in the background. 
The light intensity of the background was not exactly uniform. However, by using the p-value to measure the relative light intensity, the calibration in the central region of the tank was found to be not significantly different from the calibration obtained from elsewhere in the tank. Therefore, the dye concentration in the turbulent flow presented here was calculated based on the calibration curve obtained from a small area located in the central region of the tank.

\section{CONFINEMENT FORMULATION}

Without consideration of the dynamical dependence of the flow on buoyancy and friction forces, turbulent motion is expected to be affected only by the kinematic constraint of the side walls (i.e. zero velocity). The energy cascade process in quasi two dimensional turbulent flow of shallow depth is expected to be different from the process in unconfined threedimensional turbulent motion. Vortex stretching, the dominant mechanism in three dimensional turbulence, is absent from the process in two-dimensional motion. The kinematics of the confinement effect on the turbulent flow of shallow depth has been the subject of a number of recent investigations, [1], [2], and [4]. The results, however, were not conclusive. It is hoped that works like this go directed to this goal.

\section{FRICTION EFFECT}

While the confinement effect may be negligible, the viscosityeffect is not. Thisfluid "friction" effect suppresses large-scale motion. The results are smaller entrainment rate and subsequent higher dye concentration. The limiting case of the jet is of considerable significance. In the present context, a free jet refers to the case when friction effect is negligible. We may speak of free jets of two kinds. The free jet of the first kind (often referred to as a plane jet) is unconfined and free of friction effect but, to the best our knowledge, there have not been any experiments conducted for the starting free jet of the first kind. The jets in test TW are free jets of the second kind; the jets in this test are confined but the friction effect is again negligible.

The friction, that is the momentum sink effect, is calculated through the Law of the Wall which implies that the average velocity of a turbulent flow at a certain point is proportional to the logarithm of the distance from that point to the "wall", or the boundary of the fluid region. This law of the wall which was first published by Theodore von Karman, in 1930 [3], is only technically applicable to parts of the flow that are close to the wall, which means that it is located at a distance lower than $20 \%$ of the height of the flow.

The Law of the wall is written as:

$$
\mathrm{u}^{+}=1 / \kappa * \ln \mathrm{y}^{+}+\mathrm{C}^{+}
$$

where $\kappa$ is the Von Kármán constant and $\mathrm{y}^{+}=$is the wall coordinate, i.e., the dimensionless distance $y$ to the wall in terms of the kinematic viscosity, $v$, and the friction velocity, $\mathrm{u}_{\mathrm{t}}$, related to the wall shear, $\tau$, as follows:

$$
\mathrm{y}+=\mathrm{y} \quad \mathrm{u}_{\tau} \mathrm{v}
$$

$$
\begin{array}{r}
u^{+}=u / u_{\tau} \\
u_{t}=\sqrt{ }(\tau / \rho)
\end{array}
$$

It means that the equivalence of the friction factor is related to the iterative calculation of wall shear $\tau$ for the cell adjacent to the wall. In this particular case, the wall is assumed to be "smoothed" because they are made of glass.

\section{MATHEMATICAL FORMULATION}

The problem to be simulated is described by the injection of dye into a tank filled with water. Dimensions of the tank, location of the injection port, as well as hydrodynamic parameters of the jet are mentioned in section III defined as experiment equipment.

The mathematical representation of this problem may be considered from two different points of view; one where steady state conditions may be assumed, considering a very wide tank in such a way that the mass of water contained in the tank is much bigger than the mass injected, or through the consideration of a transient flow where mass accumulation is very small and there is no need to include compressibility effects. In this work it was adopted the latter.

The set of partial differential equations describing the problem examined herein, are those of continuity and momentum together with an additional couple of turbulence transport equations; additionally, to deal with the dye injection, a species equation is considered.

This set of equations can be expressed in a general form as follows:

$$
\frac{\partial}{\partial t}(\rho \phi)+\Delta \bullet(\rho \bar{V} \phi)=\Delta \bullet\left(\Gamma_{\phi} \Delta \phi\right)+S_{\phi}
$$

where t denotes time, $\phi$ represents any dependent variable, $V$ the velocity vector, $\rho$ the fluid density, $\Gamma$ the transport coefficient of the dependent variable, $S$ the source of $\phi$ per unit volume and $\Delta$ is the vector differential operator.

The turbulence model employed is the standard $\mathrm{k}-\varepsilon$ model, where the kinetic energy $\mathrm{k}$, and its dissipation rate $\varepsilon$, represent the velocity and length scales of the turbulent motion respectively. The turbulencemodel coefficients $\mathrm{C} \mu, \mathrm{Cd}, \mathrm{C}_{1 \varepsilon}$ and $\mathrm{C}_{2 \varepsilon}$ are assigned the constant values of $0.5478,0.1643,1.44$ and 1.92 respectively as recommended bay Launder and Spalding [8].

The dependent variables and their corresponding transport equations, are listed below in Table III, where $\mathrm{u}, \mathrm{v}$ are the velocity components in the coordinate directions $\mathrm{x}$ and $\mathrm{y}$ respectively, $\mathrm{k}$ is the turbulent kinetic energy and $\varepsilon$ its dissipation rate; $\mu_{l}$ is the laminar dynamic viscosity, $\mu_{t}$ the turbulent viscosity, and $\mu_{e}$ the effective viscosity; and $\sigma_{\varepsilon}$ are the standard turbulence-model coefficients equal to 1 and 1.314 . 
TABLE III. TRANSPORT COEFFICIENTS FOR VARIABLE

\begin{tabular}{|c|c|c|}
\hline Variable & $\phi$ & $\Gamma_{\phi}$ \\
\hline Continuity & 1 & 0 \\
\hline Momentum & $\mathrm{u}, \mathrm{v}$ & $\mu_{e}$ \\
\hline $\begin{array}{c}\text { Turbulent kinetic } \\
\text { energy }\end{array}$ & $\mathrm{k}$ & $\left(\mu_{l}+\mu_{t} / \sigma_{k}\right)$ \\
\hline $\begin{array}{c}\text { Dissipation rate } \\
\text { of } \boldsymbol{k}\end{array}$ & $\varepsilon$ & $\left(\mu_{l}+\mu_{t} / \sigma_{\varepsilon}\right)$ \\
\hline
\end{tabular}

The source term for the momentum equation is given by:

$$
S_{m}=-\underline{\Delta} p+\rho \underline{g}+\underline{\Delta} \cdot\left[\mu_{e}(\underline{\Delta} \underline{U})^{\mathrm{T}}-\frac{2}{3}\left(\mu_{e} \underline{\Delta} \bullet \underline{U}\right) I\right]^{(6)}
$$

where $\rho$ is static pressure, g gravity vector, I the unit tensor, and the superscript $\mathrm{T}$ denotes the transpose of the dyadic. are:

The corresponding source terms for the turbulence model

$$
\begin{array}{r}
S_{k}=\left(P_{k}-\rho \varepsilon+G_{B}\right)(7) \\
S_{\varepsilon}=\left(C_{1 \varepsilon} P_{k}-C_{2 \varepsilon} \rho \varepsilon+C_{3 \varepsilon} G_{B}\right) \frac{\varepsilon}{k}(8)
\end{array}
$$

where $P_{k}$ is the production rate of $k$, and $G_{B}$ is the production or destruction of $k$ due to buoyancy effects, which in this case is negligible. The production rate may be written as:

$$
P_{k}=\mu_{t}\left[\underline{\nabla} \underline{U}:\left(\underline{\nabla} \underline{U}+(\underline{\nabla} \underline{U})^{\mathrm{T}}\right)\right](9)
$$

The turbulent viscosity is calculated from the local values of $\mathrm{k}$ and $\varepsilon$ as follows:

$$
\mu_{t}=C_{\mu} \rho k^{2} / \varepsilon
$$

As mentioned above, the model takes into account the friction effect at solid walls through the wall-function approach outlined by Rodi [13], which means that the boundary conditions are not specified right at the wall but at a point outside the viscous sublayer, where the logarithmic law of the wall prevails and the turbulence can be assumed in local equilibrium.

The set of partial differential equations are solved using a Finite Volume Scheme and the solution algorithm is based upon the well-known iterative guess-and-correct procedure of Patankar and Spalding [11], modified according to the SIMPLEST algorithm of Spalding [14].The usual friction term that appears in them is solved iteratively through the momentum sink. This method is more precise that the empirically based one for the aforementioned friction term.

\section{SHALLOW WATER EQUATIONS}

It is generally accepted that the unsteady flow of water in a two-dimensional space may be described by the shallow water equations, which represent mass and momentum conservation and can be obtained by depth averaging the Navier-Stokes equations in the vertical direction. This leads to a 2D formulation in terms of depth averaged quantities and the water depth itself, they form the following system of equations:

$$
\begin{array}{r}
\frac{\partial h}{\partial t}+\frac{\partial h u}{\partial x}+\frac{\partial h v}{\partial y}=0,(11) \\
\frac{\partial h v}{\partial t}+\frac{\partial h u^{2}}{\partial x}+\frac{\partial h u v}{\partial y}=f h v+h \tau_{s x}-g h \frac{\partial H}{\partial x}+c f u \sqrt{u^{2}+v^{2}},(12) \\
\frac{\partial h v}{\partial t}+\frac{\partial h u^{2}}{\partial x}+\frac{\partial h u v}{\partial y}=-f h v+h \tau_{s x}-g h \frac{\partial H}{\partial x}+ \\
c f u \sqrt{u^{2}+v^{2}},(13)
\end{array}
$$

$\mathrm{f}$ represents the Coriolis parameter $\mathrm{f}=2 \Omega \sin \varphi$ and contributes as a non-inertial volumetric force when geophysical problems of planetary scale are considered. It contains the effect of the Earth rotation on a moving fluid ( $\Omega$ being the rotation angular velocity and $\varphi$ being the geographic latitude). The relative importance of this term is controlled by the Rosby number. The shear stresses acting on the free surface are due to viscosity and the dynamic boundary condition requires that they are continuous across the surface, that is, their value at the internal part is equal to the external value imposed by the wind. This is the way to include the effect of the wind and is usually modeled using

$$
\tau_{s}=p c_{w} W^{2},(14)
$$

asemiempirical formula where $\mathrm{W}$ is the module of the wind velocity and $c_{w}$ is a coefficient depending on the wind direction. Both the magnitude and direction of the wind force are determined by the atmospheric flow. The coefficient $c f$ appearing in the friction term is normally expressed in terms of the Manning $n$ or the Chezy roughness factor

$$
\begin{aligned}
& c f u \sqrt{u^{2}+v^{2}}=\frac{n^{2} u \sqrt{u^{2}+v^{2}}}{h^{\frac{4}{3}}},(15) \\
& c f v \sqrt{u^{2}+v^{2}}=\frac{n^{2} v \sqrt{u^{2}+v^{2}}}{h^{\frac{4}{3}}}(16)
\end{aligned}
$$

The roughness coefficient $n$ is in principle dependent on the nature of boundary solid surfaces, but also on the flow Reynolds number, although the latter factor is normally neglected.

The terms originated from the depth average of the pressure gradient are $g \partial H / \partial x, g \partial H / \partial y$, which, using $H=h+z_{b}$, can be written as

$$
g \frac{\partial H}{\partial x}=g \frac{\partial h}{\partial x}+g \frac{\partial z_{b}}{\partial x}, g \frac{\partial H}{\partial y}=g \frac{\partial h}{\partial y}+g \frac{\partial z_{b}}{\partial y}(17)
$$

The bottom level variations are expressed in the form of a slope as

$$
S_{0 x}=-\frac{\partial z_{b}}{\partial x}, S_{0 y}=-\frac{\partial z_{b}}{\partial y}(18)
$$

And the same notation is applied to the friction terms, using the energy grade slopes.

$$
S_{f x}=\frac{c_{f} u \sqrt{u^{2}+v^{2}}}{g h}, \quad S_{f y}=\frac{c_{f} v \sqrt{u^{2}+v^{2}}}{g h}(19)
$$




\section{PRESENTATION AND DISCUSSION OF RESULTS}

Two dimensional transient calculations were performed. Several grid sizes were tested until a 69 x 55 non uniform mesh with 3,795 cells distributed along $\mathrm{X}$ and $\mathrm{Y}$ showed grid independence of results. The distribution of the mesh is shown in figure 4, to represent the transient of 35 seconds, i.e. 350 intervals of 0.1 seconds each were used.

To compare the results with the experimental photographs previously shown, the distance of the jet front and some geometric factors of the jet were taken into consideration. For the time intervals of 3 and 8 seconds, the jet does not reach the bottom of the tank, and from the experiments it may be determined that its advance front locates at approximately 0.53 and $0.83 \mathrm{~m}$ from the injection point. For those times, the simulations situate the jet fronts at approximately 0.54 and 0.85 $\mathrm{m}$, which is 1.3 and $1.6 \%$ farther than the experimental results. Figures $5 \mathrm{a}-\mathrm{b}$ and $6 \mathrm{a}-\mathrm{b}$ make a direct comparison of the envelope of the jet for 3 and 8 seconds after injection.

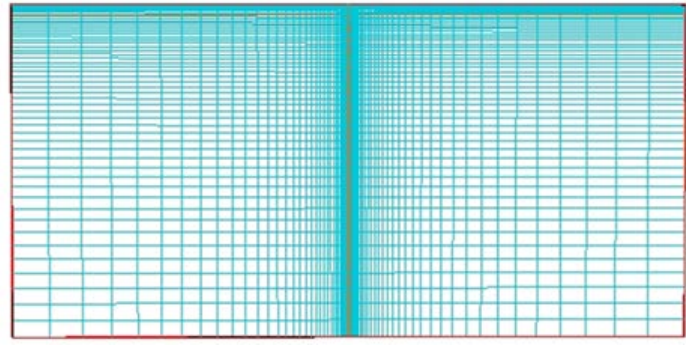

Fig. 4.Cells distribution.

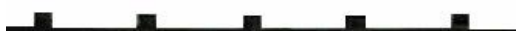

(a)

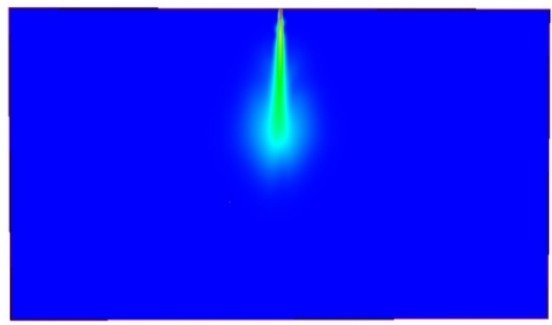

(b)
Fig.5.Jet position after 3 seconds of injection. (a) experimental and (b) numerical.

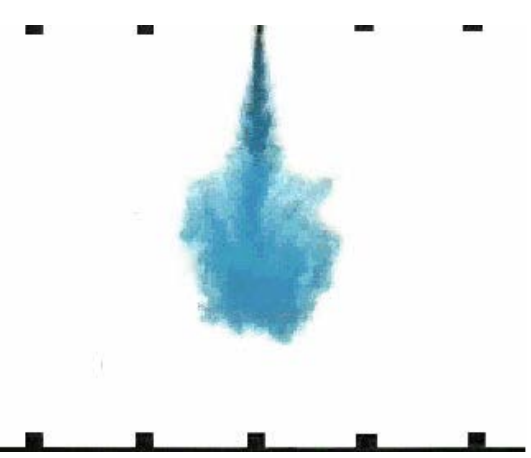

(a)

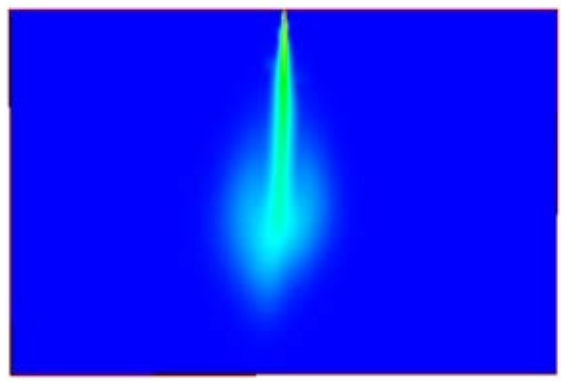

(b)

Fig. 6.Jet position after 8 seconds of injection. (a) experimental and (b) numerical.

After 18 seconds of injection, the dye touches the bottom and starts spreading without reaching the lateral walls. As time goes on, the flow accentuates more and more its asymmetry with respect to the injection axis, showing a larger entrainment on the left side of the jet. This can be noticed in the photograph as a small white path; the numerical results managed to pick up this asymmetry and the entrainment is shown as a darker blue path. To compare the results, it was determined the position of the jet shoulder; for the right side of the jet, the experiment indicates a distance of approximately $0.44 \mathrm{~m}$ from the bottom of the tank, while the numerical calculations gave approximately $0.48 \mathrm{~m}$, that is $10.3 \%$ higher. Both cases are shown in figure 7. 


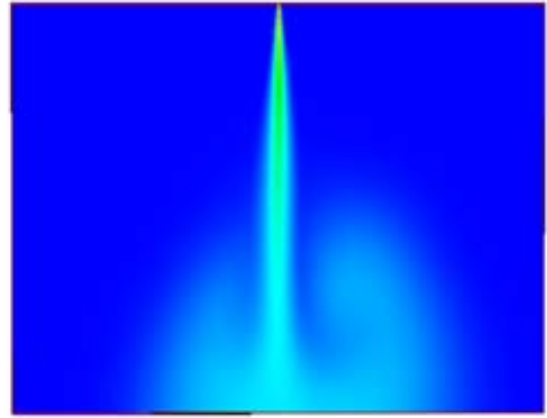

(b)

Fig.7.Jet position after 18 seconds of injection. (a) experimental and (b) numerical.

Thirty five seconds after injection, the jet reaches the lateral walls of the container and starts climbing up. Experimentally the dye goes up approximately $0.63 \mathrm{~m}$, and numerically locates $0.70 \mathrm{~m}$ above the bottom of the tank, i.e., $11.7 \%$ more. Figure 8 presents both cases.

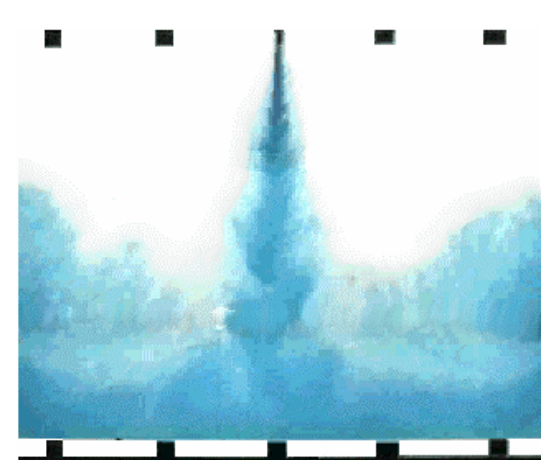

(a)

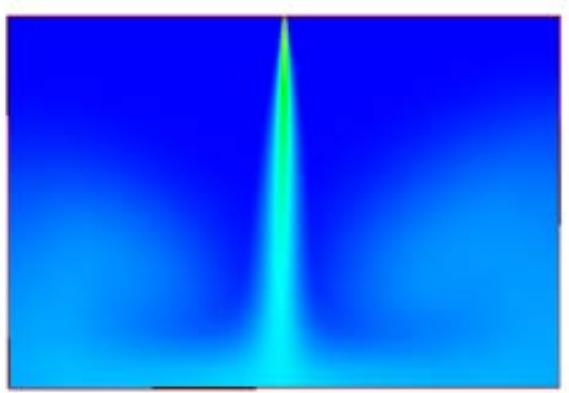

(b)

Fig.8.Jet position after 35 seconds of injection. (a) experimental and (b) numerical.

Table IV summarizes the comparisons between the experimental and the numerical results.
TABLE IV. EXPERIMENTAL AND NUMERICAL RESULTS

\begin{tabular}{|l|c|c|c|c|}
\hline \multicolumn{1}{|c|}{ Jet } & $\begin{array}{c}\text { Time } \\
\text { (sec) }\end{array}$ & $\begin{array}{c}\text { Distance } \\
\text { exp. (m) }\end{array}$ & $\begin{array}{c}\text { Distance } \\
\text { num. (m) }\end{array}$ & $\begin{array}{c}\text { Differen } \\
\text { ce (\%) }\end{array}$ \\
\hline $\begin{array}{l}\text { Distance of front } \\
\text { from top }\end{array}$ & 3 & 0.5339 & 0.5411 & 1.34 \\
\hline $\begin{array}{l}\text { Distance of front } \\
\text { from top }\end{array}$ & 8 & 0.836 & 0.8495 & 1.62 \\
\hline $\begin{array}{l}\text { Distance of shoulder } \\
\text { from bottom }\end{array}$ & 18 & 0.4422 & $0-4879$ & 10.31 \\
\hline $\begin{array}{l}\text { Distance over wall } \\
\text { from bottom }\end{array}$ & 35 & 0.6302 & 0.704 & 11.70 \\
\hline $\begin{array}{l}\text { Distance of front } \\
\text { from top }\end{array}$ & 3 & 0.5339 & 0.5411 & 1.34 \\
\hline
\end{tabular}

\section{CONCLUSIONS}

A two dimensional transient calculation was performed to represent the injection of a dye into a rectangular container. A comparison of the numerical results with the experimental data showed a very good agreement in terms of the position reached by the jet at different times after injection. It would be advisable to make a comparison in terms of the dye concentration employing different turbulence models intending to get even better results. Further stages in this study contemplate the use of a two fluid model to take into account the density difference.

\section{REFERENCES}

[1] Chu, V.H. and Babarutsi, S. (1988) "Confinement and bed-friction effects in shallow turbulent mixing layers,” J. of Hydraulics Engineering, ASCE. Vol. 114, pp. 1257-1274.

[2] Chu, V.H. and Babarutsi, S. (1989) "A k- $\varepsilon$ model for depth-averaged flow calculation," Proc. $3^{\text {rd }}$ National Conference on Hydraulic Engineering, pp. 999-1004.

[3] von Kármán, Th. (1930), "Mechanische Ähnlichkeit und Turbulenz", Nachrichten von der Gesellschaft der Wissenschaften zu Göttingen, Fachgruppe 1 (Mathematik)5: 58-76.

[4] Chu, V.H. and Baines, W.D. (1989) "Entrainment by a buoyant jet between confined walls" J. of Hydraulics Engineering, ASCE. Vol. 115, No. 4, pp. 475-492.

[5] Chu, V. H., Wu, J. H., and Khayat, R. E. (1991) "Stability of transverse shear flows in shallow open channels,” J. of Hydraulics Engineering. Vol. 117, pp.1-19.

[6] Babarutsi, S., and Chu, V.H. (1991) "A two length-scale model for quasi-two-dimensional turbulent shear flows," Proc. of the 24th IAHR Congress, Madrid, Vol. C, pp. 51-60.

[7] Booij, R. (1989) "Depth-average k- $\varepsilon$ modeling" Proc. 23 $3^{\text {rd }}$ IAHR Congress, Ottawa, Vol. A, pp. 199-206.

[8] Launder, B.E., Spalding, D.B. (1974). “The Numerical Computation of Turbulent Flow”, Comp. Methods Applied Mechanical Engineering, 269,289.

[9] Madsen, P., Rugbjerg, M. and Warren, I.R. (1988) "Subgrid modeling in depth integrate flows," $21^{\text {st }}$ Int'l conf. on Coastal Engrg., ASCE, pp. 505-511.

[10] Nadaoka, K., and Yagi, H. (1993) "Horizontal large-eddy computation of river flow transverse by SDS / 20H model,” J. of Hydraulics, Coastal and Environmental Engineering, JSCE, No. 473, pp. 35-44 (in Japanese).

[11] Patankar, S.V., Spalding, D.B. (1972). “A calculation procedure for heat, mass and momentum transfer in three-dimensional parabolic flows”, Int. J. Heat and Mass Transfer, 15, 1787-1805. 
[12] Rastogi, A. K. and Rodi, W. (1978) "Predictions of heat and mass transfer in open channels," J. Hydraulic Engineering, Vol. 104, pp. 397420.

[13] Rodi, W. (1980). Turbulence Models and their Applications in Hydraulics - A State of the Art Review. Book Pub., Delft, The Netherlands.

[14] Spalding, D.B. (1982). Four lectures on the PHOENICS computer Code, CFD/82/5, CFDU, Imperial College, University of London.. Creative Commons Attribution License 4.0
(Attribution 4.0 International, CC BY 4.0)

This article is published under the terms of the Creative Commons Attribution License 4.0

https://creativecommons.org/licenses/by/4.0/deed.en US 